\title{
Neighbours hiding in the Galactic plane, a new $M / L$ dwarf candidate for the 8 pc sample
}

\author{
R.-D. Scholz \\ Leibniz-Institut für Astrophysik Potsdam (AIP), An der Sternwarte 16, 14482 Potsdam, Germany \\ e-mail: rdscholz@aip.de
}

Received 8 November 2013 / Accepted 12 December 2013

\section{ABSTRACT}

\begin{abstract}
Aims. Using Wide-field Infrared Survey Explorer (WISE) data and previous optical and near-infrared sky surveys, we try to identify still missing stellar and substellar neighbours of the Sun.

Methods. When checking the brightest red WISE sources for proper motions and colours expected for nearby M and L dwarfs, we also approached the thin Galactic plane. Astrometry (proper motion and parallax measurements) and the available photometry were used to obtain first estimates of the distance and type of nearby candidates.

Results. We have discovered WISE J072003.20-084651.2, an object with moderately high proper motion $(\mu \approx 120$ mas/yr) that lies at low Galactic latitude $\left(b=+2.3^{\circ}\right)$, with similar brightness $(J \approx 10.6, w 2 \approx 8.9)$ and colours $\left(I-J \approx 3.2, J-K_{\mathrm{s}} \approx 1.2, w 1-w 2 \approx 0.3\right)$ as the nearest known M-type brown dwarf LP 944-20. With a photometric classification as an M9 \pm 1 dwarf, its photometric distance lies in the range between about 5 and 7 pc, based on comparison with absolute magnitudes of LP 944-20 alone or of a sample of M8-L0 dwarfs. The slightly larger distance derived from our preliminary trigonometric parallax ( $7.0 \pm 1.9 \mathrm{pc})$ may indicate a close binary nature. The new neighbour is an excellent target for planet search and low-mass star/brown dwarf studies.
\end{abstract}

Key words. astrometry - proper motions - stars: distances - stars: kinematics and dynamics - brown dwarfs - solar neighborhood

\section{Introduction}

The incomplete knowledge of our nearest stellar neighbours, demonstrated, e.g., by Henry et al. (1997), has motivated many astronomers to take part in the search for still missing late-type dwarfs and white dwarfs surrounding the Sun. The 74 AFGK stars within $10 \mathrm{pc}$ have already been known for a long time and their trigonometric parallaxes were measured by HIPPARcos (ESA 1997; van Leeuwen 2007). In contrast, the numbers of M dwarfs and white dwarfs increased from 198 to 248 and from 18 to 20, respectively, during the last 12 years, according to the Research Consortium on Nearby Stars (RECONS) ${ }^{1}$. This progress was achieved thanks to many individual discoveries and subsequent time-consuming trigonometric parallax measurements of the new candidates. The RECONS error requirement for the parallax of less than 10 mas means that the distance for every RECONS 10 pc sample member is known to better than $10 \%$. As of 1 January 2012, there are also five L and ten $\mathrm{T}$ dwarfs falling in the RECONS $10 \mathrm{pc}$ sample. Only the nearest 100 systems $(d<6.7 \mathrm{pc})$ of this high-quality sample are published on the RECONS Web site.

An updated 8 pc sample, which includes objects with less accurate and photometric distance estimates, was compiled by Kirkpatrick et al. (2012). This sample was used to explore the field brown dwarf mass function, but most of the $22 \mathrm{~T}-$ and 8 Y-type brown dwarfs listed still need higher accuracy to meet the RECONS requirement. Only three L dwarfs are known within $8 \mathrm{pc}$, and all these are late-L types. Late-M and L dwarfs may be stellar or substellar depending on their age, and lithium absorption can be used as a distinguishing criterion (Rebolo et al. 1992). Among six late-M (>M8) dwarfs in the 8 pc sample of

\footnotetext{
http://www.chara.gsu.edu/RECONS/
}

Kirkpatrick et al. (2012), there is one M9 dwarf (LP 944-20) that was correspondingly classified as a relatively young (a few hundred Myr compared to several Gyr typical for the solar neighbourhood) brown dwarf (Tinney 1998; Ribas 2003; Pavlenko et al. 2007).

The Galactic plane, a region often excluded or considered as problematic in previous searches due to image crowding, is probably still hiding many close neighbours, mainly cool stars, and even cooler brown dwarfs. Some recent large-area searches successfully approached (Looper et al. 2008) or included (Phan-Bao et al. 2008; Folkes et al. 2012; Luhman 2013) the Galactic plane. Dedicated Galactic plane surveys (Lucas et al. 2010; Burningham et al. 2011; Beamín et al. 2013) have also succeeded in finding new neighbours.

Here, we introduce a previously missing probable $8 \mathrm{pc}$ sample member in the Galactic plane, WISE J072003.20-084651.2 (hereafter WISE J0720-0846), which was identified among bright candidates selected from Wide-field Infrared Survey Explorer (WISE; Wright et al. 2010) data (Sect. 2). Despite its relatively small proper motion (Sect. 3 ), the preliminary trigonometric parallax, based on the available 12 epochs of observations (Sect. 3), leads to a distance of about 7 pc. Its magnitudes and colours, which are very similar to those of the M9 dwarf LP 944-20, place it at a photometric distance of about 5-7 pc (Sect. 4).

\section{Candidate search and cross-identification}

Our search strategy aimed at hidden M- and L-type low-mass stellar and substellar neighbours of the Sun. They appear very bright in the mid-infrared WISE survey and can be separated from distant reddened stars and red giants by their characteristic optical-to-infrared colours and high proper motions (HPMs; 
see, e.g., Phan-Bao et al. 2008). To measure these properties, a cross-identification with their correct counterparts in nearinfrared and optical surveys is necessary. Our idea was to check this cross-identification for every bright WISE source with WISE colours expected for dwarfs of spectral type $\geq$ M5 (see Kirkpatrick et al. 2011). This is, therefore, a combined HPM and colour-based search restricted to the brightest and hence nearest M/L dwarfs. In comparison to other HPM surveys such as, e.g., SUPERBLINK (started by Lépine et al. 2002) and SuperCOSMOS-RECONS (started by Hambly et al. 2004), this is a small survey (or completeness check) dedicated to a certain class of objects in the solar neighbourhood.

To select nearby $\mathrm{M} / \mathrm{L}$ dwarf candidates, we applied the following selection criteria to the WISE All-Sky source catalogue:

1. $w 1-w 2 \geq 0.2$ and $w 2<10$ (compare with Figs. 1 and 29 in Kirkpatrick et al. 2011);

2. $w 2-w 3<2.5$ and $w 3-w 4<2.0$ (similar colour cuts were described by Luhman 2013, for excluding extragalactic sources).

All WISE sources (including point and extended sources) were considered as $\mathrm{M} / \mathrm{L}$ dwarf candidates if they matched the criteria listed above. After pre-selecting the $\mathrm{M} / \mathrm{L}$ dwarf candidates,we performed two different searches for nearby HPM objects among them using the cross-identification with the Two Micron All Sky Survey (2MASS; Skrutskie et al. 2006) listed in the WISE AllSky source catalogue.

First (3a), a WISE M/L candidate was flagged as a HPM candidate if there was no 2MASS source within 3 arcsec of this WISE candidate. This all-sky search was sensitive to all red WISE objects brighter than $w 2=10$ with proper motions larger than about 300 mas/yr because the typical epoch difference between 2MASS and WISE is about ten years.

In our second search (3b), we focused on stars with $|b|>$ $2^{\circ}$ to exclude the very thin Galactic plane and considered all WISE M/L candidates as possible HPM objects if there was a 2MASS source between 1 arcsec and 3 arcsec radius of their WISE position. This search was sensitive to stars with proper motions between about 100 and 300 mas/yr.

The resulting numbers of candidates, without and with 2MASS counterparts in the WISE catalogue (search (3a) and (3b), respectively), were in each case about 9500. These two samples were then cross-identified with known SIMBAD objects within a search radius of 60 arcsec using the cross-match service provided by the Centre de Données astronomiques de Strasbourg (CDS). All candidates not identified with previously known nearby or HPM objects were cross-checked in the DwarfArchives in addition (Gelino et al. 2012), and, also visually inspected with the help of the IRSA Finder Charts tool ${ }^{2}$ providing Digitised Sky Surveys (DSS), 2MASS, and WISE images for a given object at a glance.

Among the candidates of search (3a), the majority turned out to be false HPM detections, whereas we found about 1000 known HPM, but only about ten new HPM objects. The latter will be published elsewhere. Their estimated photometric distances lie in the range between 10 and $30 \mathrm{pc}$. The nearest known objects from the 8 pc sample of Kirkpatrick et al. (2012) were used for checking the success rate of this first search. Among the late-M ( $\geq$ M5) dwarfs in the 8 pc sample, there are 44 that have no close companions of earlier spectral type and proper motions larger than 300 mas/yr. Six of them have $0.15<w 1-w 2<0.2$,

\footnotetext{
2 http://irsa.ipac.caltech.edu/applications/ finderchart/
}

as can be expected due to the spread seen in Fig. 1 of Kirkpatrick et al. (2011), whereas two have even negative colour indices due to an overlap with background stars. The remaining 36 objects were included in our first candidate list and also re-discovered as HPM objects so that the success rate for the late-M dwarfs of the 8 pc sample was $82 \%$. All three late-L dwarfs in the 8 pc sample (L5, L8, and L9) are fainter $(10<w 2<11)$ than the survey limit set by condition 1 .

The success rate for the second search (3b), for moderately HPM objects, is difficult to estimate. The reasons are a) there are only three known $\geq$ M5 dwarfs (without close companions of earlier spectral type) with proper motions below 300 mas/yr in the 8 pc sample, and b) this second search is still ongoing. However, it already led to an intriguing new bright red $(J=10.6$, $\left.J-K_{\mathrm{s}}=1.2, J-w 2=1.8\right)$ candidate at very low Galactic latitude $\left(b=+2.3^{\circ}\right)$. Its proper motion was clearly visible as the optically fainter counterpart on the red DSS1 $(E)$ plate was shifted by a few arcseconds with respect to the 2MASS position. We extracted the multi-epoch positions measured on four plates $\left(E, B_{J}, R\right.$, and $\left.I\right)$ within the SuperCOSMOS Sky Surveys (SSS; Hambly et al. 2001) together with two more positions ( $R H a$ and $S R 2$ ) measured in the SuperCOSMOS $\mathrm{H}_{\alpha}$ survey (Parker et al. 2005). The object was also detected with a large colour index $I-J \approx 3.2$ in the DEep Near-Infrared Survey (DENIS; Epchtein et al. 1997), and we extracted positional measurements (and an $r^{\prime}$ magnitude) from the Carlsberg Meridian Catalog (CMC, 2006; Table 1) that helped to increase the parallax factor coverage.

\section{Proper motion and parallax}

Proper motion measurements of WISE J0720-0846 were already listed in the USNO B1.0 (Monet et al. 2003) and PPMXL (Röser, Demleitner \& Schilbach 2010) catalogues. The B1.0 catalogue lists $(-36,-102) \pm(13,27) \mathrm{mas} / \mathrm{yr}$, whereas the PPMXL values are more precise: $(-39,-107) \pm(7,7) \mathrm{mas} / \mathrm{yr}$. From the collected 12 epochs (Table 1), We expected to further improve the proper motion and also aimed at a preliminary parallax measurement. For this purpuse, we used the software of Gudehus (2001) allowing for a combined proper motion and parallax solution with weights corresponding to the positional errors of the different surveys. Before that, we computed the mean offsets of some reference stars around the target with respect to their 2MASS coordinates to bring the different positional measurements of the target to a common system, A similar procedure was also applied by Luhman (2013) before he determined the parallax and proper motion of the very close $\left(d_{\text {trig }}=2.0 \pm 0.15 \mathrm{pc}\right)$ new neighbour of the Sun, WISE J104915.57-531906.1, using its DSS, DENIS, 2MASS, and WISE positions.

Suitable reference stars were selected within 3 arcmin of the 2MASS position of WISE J0720-0846 if they:

- appeared as isolated, well-measured objects on/in each of the 12 plates/surveys;

- had high-quality flags (AAA) and $J<16$ in 2MASS,

- had no significant proper motion in the PPMXL (<10 mas/yr); and

- were not too bright or too faint in the WISE post-cryo data (large positional spread of their inividual exposures).

With these conditions we were left with nine reference stars (Fig. 1), out of 17 possible stars before applying the last criterion. This figure also shows the changes in resolution and brightness of the stars with the different passbands. All coordinates 
R.-D. Scholz: New M/L dwarf candidate for the 8 pc sample

Table 1. Multi-epoch positions, their uncertainties, applied corrections (offsets), and photometry of WISE J0720-0846.

\begin{tabular}{|c|c|c|c|c|c|c|c|}
\hline $\begin{array}{l}\alpha(\mathrm{J} 2000) \\
\left({ }^{\circ}\right)\end{array}$ & $\begin{array}{l}\delta(\mathrm{J} 2000) \\
\left(^{\circ}\right)\end{array}$ & $\begin{array}{c}\sigma_{\alpha, \delta} \\
(\mathrm{mas})\end{array}$ & $\begin{array}{c}\Delta_{\alpha} \\
(\mathrm{mas}) \\
\end{array}$ & $\begin{array}{c}\Delta_{\delta} \\
(\mathrm{mas})\end{array}$ & $\begin{array}{l}\text { Epoch } \\
\text { (year) }\end{array}$ & Source & $\begin{array}{l}\text { Photometry }{ }^{b} \\
\text { (mag) }\end{array}$ \\
\hline 110.014078 & -8.779181 & 240 & +20 & -307 & 1955.882 & SSS $E$ & $E_{\mathrm{pg}}=17.08$ \\
\hline 110.014088 & -8.779175 & 370 & +78 & +351 & 1955.882 & $\operatorname{DSS} O^{a}$ & \\
\hline 110.013690 & -8.779973 & 70 & +27 & +85 & 1981.188 & SSS $I$ & $I_{\mathrm{pg}}=12.91$ \\
\hline 110.013653 & -8.780144 & 220 & -16 & +119 & 1985.953 & SSS $R$ & $R_{\mathrm{pg}}=16.80$ \\
\hline 110.013642 & -8.780193 & 350 & +57 & -51 & 1990.971 & $\operatorname{SSS} B_{J}$ & $B_{J \mathrm{pg}}=19.76$ \\
\hline 110.013581 & -8.780536 & 50 & -85 & +184 & 1998.978 & DENIS & $I=13.805 J=10.674 K_{\mathrm{s}}=9.399$ \\
\hline 110.013557 & -8.780529 & 50 & 0 & 0 & 1999.142 & 2MASS & $J=10.628 H=9.919 K_{\mathrm{s}}=9.467$ \\
\hline 110.013454 & -8.780566 & 400 & +120 & -70 & 2001.005 & SSS $\mathrm{H}_{\alpha}$ & $R H a_{\mathrm{pg}}=16.11$ \\
\hline 110.013400 & -8.780524 & 500 & +51 & -25 & 2001.049 & SSS short-R & $S R 2_{\mathrm{pg}}=17.00$ \\
\hline 110.013484 & -8.780679 & 150 & +28 & +17 & 2004.066 & $\mathrm{CMC}$ & $r^{\prime}=16.850$ \\
\hline 110.013350 & -8.780903 & 60 & -6 & -2 & 2010.266 & WISE all-sky & $w 1=9.169 w 2=8.857 w 3=8.278$ \\
\hline 110.013415 & -8.780936 & 120 & -11 & -29 & 2010.792 & WISE post-cryo $^{c}$ & \\
\hline
\end{tabular}

Notes. The $\alpha, \delta$ positions of the target are already corrected for the mean offsets $\left(\Delta_{\alpha}, \Delta_{\delta}\right)$ of the reference stars with respect to their 2MASS positions. ${ }^{(a)}$ Position measured on FITS image downloaded from http://archive.stsci.edu/cgi-bin/dss_plate_finder. ${ }^{(b)}$ Photographic magnitudes marked as $\mathrm{pg}$ are accurate to about 0.1-0.2 mag. Other magnitudes listed are accurate to about 0.02 mag, except for DENIS $J$ $( \pm 0.06 \mathrm{mag})$ and $K_{\mathrm{s}}( \pm 0.09 \mathrm{mag})$, and the CCD-based CMC $r^{\prime}$ (unknown). ${ }^{(c)}$ Averaged coordinates from 14 single exposures.
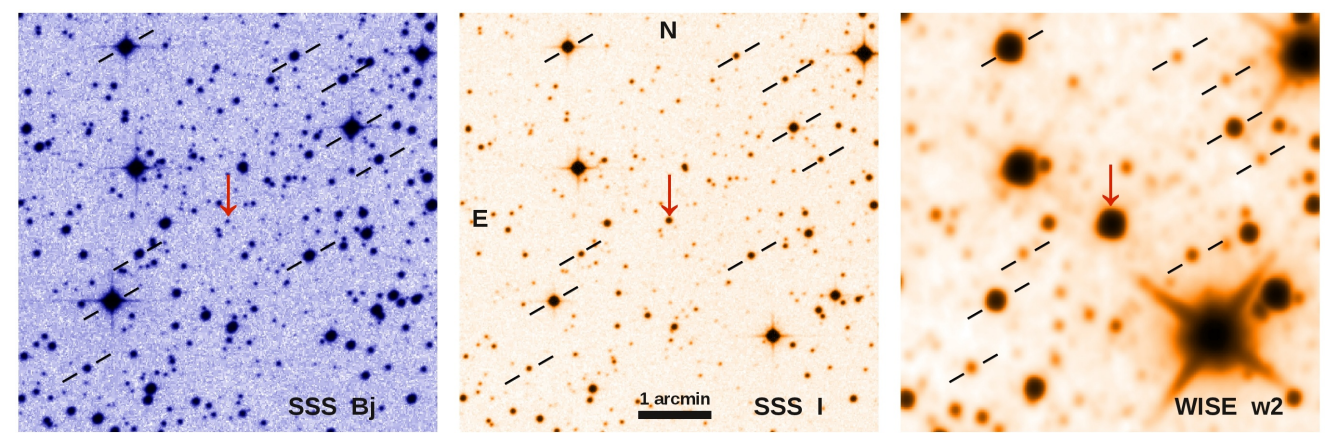

Fig. 1. Finder charts of $6 \times 6 \mathrm{arcmin}^{2}$ centred on WISE J0720-0846 from SuperCOSMOS Sky Surveys (SSS) of $B_{J}$-band (left) and $I$-band (centre) photographic plates, and from WISE $w 2$-band (right) observations. The target is marked by an arrow, and nine reference stars used for positional corrections (Sect. 3) are marked by dashes, respectively.

given in Table 1 are already corrected for the mean offsets $\Delta$ of the reference stars, also shown in the table. Some of these offsets were relatively large, as one can see, e.g., for SSS $E$ and DSS $O$, but also for DENIS. The originally measured declination values on the SSS $E$ and DSS $O$ plates were strongly deviating, but the corrected values are in good agreement.

The standard deviations of the positional differences of the reference stars vary between 20 and 80 mas for DENIS, CMC, SSS I, and WISE, whereas for the remaining red and blue photographic plate measurements from SSS and DSS the standard deviations increase to 100-250 mas. As the latter values are much larger than the effect of differential colour refraction (Deacon et al. 2005), we did not attempt to correct the target positions for these systematic errors. The uncertainties $\sigma_{\alpha, \delta}$ for the multiepoch target positions were determined based on the described standard deviations obtained from the reference stars, but they were not allowed to fall short of a lower limit of 50 mas for 2MASS. For the optical observations, we assumed larger errors for the position of the target compared to the standard deviations of the reference stars if the target appeared very faint or distorted (elliptical). Although the CMC-2MASS standard deviations of the reference stars were among the smallest, confirming the good astrometric quality of the CMC, we adopted larger $\sigma_{\alpha, \delta}$ for the CMC position of the target with a magnitude close to the CMC limit at $r^{\prime} \approx 17$. Elliptical images in the case of SSS $\mathrm{H}_{\alpha}$ and SSS short-R were the reason for assigning the largest $\sigma_{\alpha, \delta}$ to these measurements. The chosen $\sigma_{\alpha, \delta}$ for the WISE all-sky and post-cryo, 2MASS, DENIS, and SSS $R$ positions are comparable to those used by Luhman (2013). He only adopted higher values due to a blended image of his target in the case of his DSS I position.

The derived proper motion and parallax is provided in Table 2. The (relatively poor) quality of the combined fit is illustrated in Fig. 2, where the displacements of the observed positions relative to the fit are shown. The parallax error is of the same order as that derived by Luhman (2013) with input data of similar quality. However, it is larger than the error measured by Scholz et al. (2012) for another new neighbour, WISE J025409.45+022359.1, for which more accurate input data and more epochs were available. Due to the smaller parallax of WISE J0720-0846 the error leads to a much larger relative error in the distance (27\% instead of $7.5 \%$ for Luhman's object). This trigonometric parallax can only be considered as a very preliminary result as it was obtained from very different surveys.

\section{Photometric classification and distance}

For comparison of the colours of WISE J0720-0846 with those of other objects (bottom part of Table 3), first we selected the nearest known (with distances based on trigonometric parallaxes given in the top row of Table 3 ) representatives with spectral types between M8 and L1 not known to be part of close binaries, using the compilations of Kirkpatrick et al. (2012) and Gelino et al. (2012). Note that the two nearby L0 and L1 dwarfs 


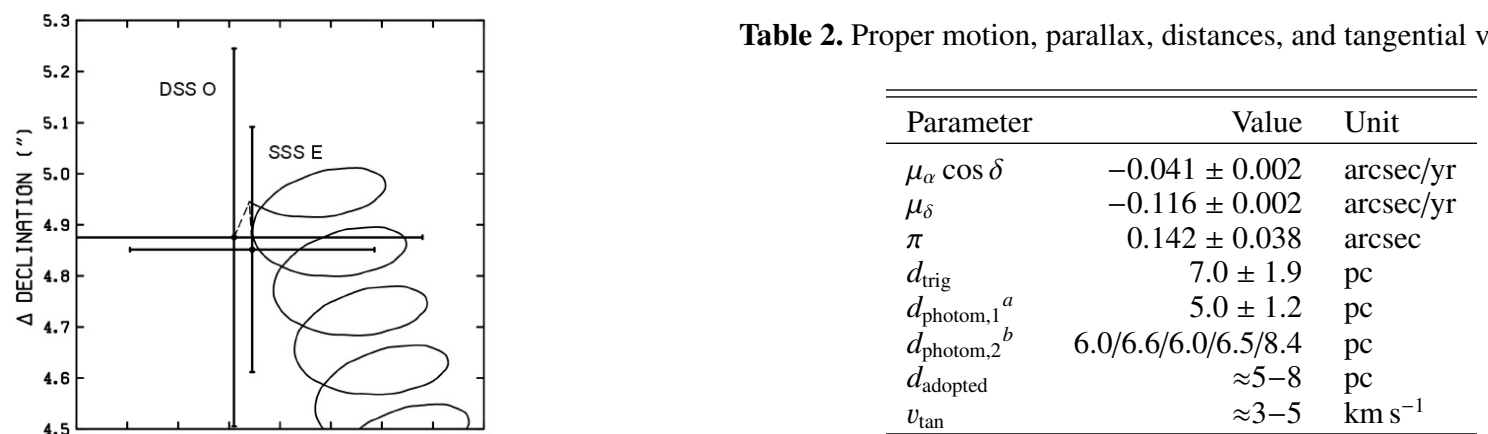

Notes. ${ }^{(a)}$ Based on comparison with absolute $J$ and $w 2$ magnitudes of LP 944-20. ${ }^{(b)}$ Applying the spectral type/absolute magnitude relations of Dupuy \& Liu (2012) to spectral types L0/M9.5/M9/M8.5/M8.

Table 3. Comparison with magnitudes/colours of nearest $\mathrm{M} / \mathrm{L}$ dwarfs.

\begin{tabular}{lcccccc}
\hline \hline Param. & $\mathrm{M}^{a}$ & ${\mathrm{M} 8.5^{b}}^{b}$ & Target & $\mathrm{M}^{c}$ & L0 $^{d}$ & L1 $^{e}$ \\
\hline$d[\mathrm{pc}]$ & 6.1 & 4.04 & & 5.0 & 27 & 14.5 \\
$J$ & 9.91 & 9.54 & 10.63 & 10.73 & 14.00 & 12.76 \\
$w 2$ & 8.25 & 7.81 & 8.86 & 8.81 & 12.09 & 10.95 \\
$M_{J}$ & 11.0 & 11.5 & & 12.2 & 11.8 & 12.0 \\
$M_{w 2}$ & 9.3 & 9.8 & & 10.3 & 9.9 & 10.1 \\
\hline$r^{\prime}-J$ & 6.07 & & 6.22 & & & 6.95 \\
$I-J$ & & 3.13 & 3.18 & 3.24 & & \\
$J-H$ & 0.68 & 0.63 & 0.71 & 0.71 & 0.79 & 0.72 \\
$J-K_{\mathrm{s}}$ & 1.14 & 1.09 & 1.16 & 1.18 & 1.32 & 1.21 \\
$J-w 2$ & 1.66 & 1.72 & 1.77 & 1.92 & 1.90 & 1.81 \\
$w 1-w 2$ & 0.22 & 0.29 & 0.31 & 0.32 & 0.25 & 0.24 \\
$w 2-w 3$ & 0.17 & 0.35 & 0.58 & 0.54 & -0.04 & 0.42 \\
\hline
\end{tabular}

Notes. The $r^{\prime}$ magnitudes are from CMC and SDSS, $I$ from DENIS, $J H K_{\mathrm{s}}$ from 2MASS, and $w 1, w 2$, and $w 3$ magnitudes are from the WISE all-sky survey. Object names, trigonometric parallaxes, and spectral type references: ${ }^{(a)}$ VB 10, $164.3 \pm 3.5$ mas (Tinney 1996), classified as M8Ve: in Henry et al. (2004); ${ }^{(b)}$ DENIS-P J104814.7-395606.1, $247.71 \pm 1.55$ mas (Jao et al. 2005), originally classified as M9 by Delfosse et al. (2001), reclassified as M8.5 by Henry et al. (2004); (c) LP 944-20, $201.4 \pm 4.2$ mas (Tinney 1996), originally typed as $>$ M9 by Tinney \& Reid (1998), reclassified as M9 by Henry et al. (2004); (d) 2MASP J0345432+254023, $37.1 \pm 0.5$ mas (Dahn et al. 2002), optical type L0 (Kirkpatrick et al. 1999), NIR type L1 \pm 1 (Knapp et al. 2004); ${ }^{(e)}$ 2MASSW J1439284+192915, 69.6 \pm 0.5 mas (Dahn et al. 2002), optical type L1 (Kirkpatrick et al. 1999).

in Table 3 are fainter than the limiting magnitude $(w 2<10)$ applied in our search (Sect. 2). For the M8 dwarf, VB 10, we found two counterparts observed at different epochs in the CMC with rather different $r^{\prime}$ magnitudes (15.549 and 16.410) and used the average in computing the $r^{\prime}-J$ colour. Only the L1 comparison object has an $r^{\prime}$ magnitude in the Sloan Digital Sky Survey (SDSS), data release 7 (Abazajian et al. 2009) that can be compared with CMC photometry. The $r^{\prime}-J$ colours are just given for completeness, but not discussed further as the CMC errors are unknown, and as there may be systematic errors at the faint end of the CMC.

WISE J0720-0846 appears to be most similar in its colours (and also apparent magnitudes listed in the second and third rows of Table 3) to the M9 brown dwarf, LP 944-20. Except for $J-w 2$, all colours of these two objects agree within $0.06 \mathrm{mag}$. The combination of colours $I-J \approx 3.2$ and $J-K_{\mathrm{s}} \approx 1.2$ is also typical of M9 dwarfs as shown in Fig. 2 of Phan-Bao (2008). However, there is an expected spread in the colour indices, and so the new object also resembles the L1 $(J-H$ and $J-w 2)$, M8 $(J-H)$ and M8.5 (w1 - w2) dwarfs. The comparison of three of the
Fig. 2. Relative astrometry of WISE J0720-0846 after conversion of the positions from DSS, SSS, DENIS, CMC, and WISE to a common system represented by 2MASS (points with error bars; see Table 1 and text) and the combined proper motion and parallax solution (curve, see Table 2). Dashed lines show the displacements of observed positions relative to the fit. The panels show (from top to bottom) observations from 1955, 1981-1990, 1998-2004, and 2010, respectively. 


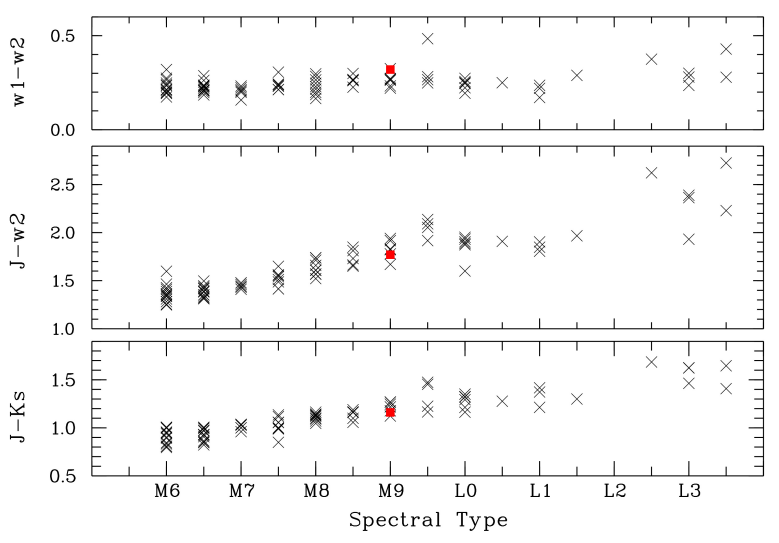

Fig. 3. Colours of single M6-L3.5 dwarfs (crosses) from Dupuy \& Liu (2012) compared to those of WISE J0720-0846 (red squares).

colours with those of a larger sample of objects listed in Dupuy \& Liu (2012) is shown in Fig. 3. Known close binaries, young and overluminous objects, as well as subdwarfs, were excluded here. Based on the comparison of colours in Table 3 and Fig. 3, we tentatively classify WISE J0720-0846 as an M9 \pm 1 dwarf.

The absolute magnitudes of the comparison objects (4th and 5th row of Table 3) show a clear trend towards fainter absolute magnitudes with later spectral types, however, this trend is interrupted by the M9 brown dwarf LP 944-20 that is even fainter than the L1 dwarf. As LP 944-20 is apparently an outlier (see also, e.g., Bihain et al. 2010), but exhibits colours very similar to WISE J0720-0846, we computed two different photometric distances. One, $d_{\text {photom, } 1}$ in Table 2 , is the average photometric distance obtained from the comparison of the apparent $J$ and $w 2$ magnitudes of WISE J0720-0846 with the corresponding absolute magnitudes of LP 944-20, assuming an uncertainty in the absolute magnitude of $0.5 \mathrm{mag}$. For a second estimate of the photometric distance $\left(d_{\text {photom, } 2}\right)$, we used the spectral type/absolute magnitude relations (for $M_{J}$ and $M_{w 2}$ ) provided by Dupuy \& Liu (2012). They rely on a larger number of typically more distant objects with measured trigonometric parallaxes. The five values of $d_{\text {photom,2 }}$ correspond to the distances as computed for types from L0 to M8. The mean value of $d_{\text {photom, } 2}$ is $6.7 \pm 0.5 \mathrm{pc}$. The small formal error shows that the systematic differences between the five sub-types are mostly smaller than the uncertainty of the absolute $J$ and $w 2$ magnitudes of M9 dwarfs of about 0.3 mag in Dupuy \& Liu (2012). If only their data for M9 dwarfs are used, the result is $6.0 \pm 0.9 \mathrm{pc}$. Although the distances obtained from the trigonometric parallax and from photometry formally agree within the errors, the photometric distance tends to be smaller. The adopted distance of $\approx 5-8 \mathrm{pc}$ is the range that is covered by all of our trigonometric and photometric estimates.

\section{Conclusions}

The new candidate for the $8 \mathrm{pc}$ sample is probably one of the nearest late-M or early-L dwarfs. It has a relatively small proper motion, but the resulting small tangential velocity is not unusual for a member of the Galactic thin disk population. Given its bright magnitude and red colour in DENIS data, it is unclear why this M9 dwarf candidate was not already found in the Galactic plane survey of Phan-Bao et al. (2008). Classification spectroscopy is needed to confirm the photometrically estimated spectral type, and medium-resolution spectroscopy will allow for the lithium test to clarify if this is a brown dwarf. Future observations will also show whether the larger distance derived from the preliminary trigonometric parallax compared to most of the photometric distance estimates may indicate that WISE J0720-0846 is, in fact, a close binary. So this is a very promising new target for high-resolution imaging, but also for trigonometric parallax and planet-search programmes, suitable for observatories in the Northern and Southern hemispheres.

Acknowledgements. This research has made use of the NASA/IPAC Infrared Science Archive, which is operated by the Jet Propulsion Laboratory, California Institute of Technology, under contract with the National Aeronautics and Space Administration, of data products from WISE, which is a joint project of the University of California, Los Angeles, and the Jet Propulsion Laboratory/California Institute of Technology, funded by the National Aeronautics and Space Administration, and from 2MASS. We have also extensively used the SIMBAD database and the VizieR catalogue access tool operated at the CDS, including the CDS xMatch service. We thank Jesper Storm and Axel Schwope for helpful discussion and the anonymous referee for her/his comments and suggestions, which helped us improve this paper.

Note added in proof. The new trigonometric parallax measured for LP 944-20 by Dieterich et al. (2014) leads to $d_{\text {photom, } 1}=6.3 \pm$ $1.5 \mathrm{pc}$ for WISE J0720-0846, which is in better agreement with $d_{\text {photom,2 }}$ and $d_{\text {trig }}$ and yields $d_{\text {adopted }} \approx 6-8$ pc.

\section{References}

Abazajian, K. N., Adelman-McCarthy, J. K., Agüeros, M. A., et al. 2009, ApJS, 182,543

Beamín, J. C., Minniti, D., Gromadzki, M., et al. 2013, A\&A, 557, L8

Bihain, G., Rebolo, R., Zapatero Osorio, M. R., Béjar, V. J. S., \& Caballero, J. A. 2010, A\&A, 519, A93

Burningham, B., Lucas, P. W., Leggett, S. K., et al. 2011, MNRAS, 414, L90

Carlsberg Meridian Catalog (CMC14) 2006, Copenhagen University Obs., Institute of Astronomy, Cambridge, UK, Real Instituto y Observatorio de la Armada en San Fernando

Dahn, C. C., Harris, H. C., Vrba, F. J., et al. 2002, AJ, 124, 1170

Deacon, N. R., Hambly, N. C., Henry, T. J., et al. 2005, AJ, 129, 409

Delfosse, X., Forveille, T., Martín, E. L., et al. 2001, A\&A, 366, L13

Dieterich, S. B., Henry, T. J., Jao, W.-C., et al. 2014, AJ, in press [arXiv: 1312.1736]

Dupuy, T. J., \& Liu, M. C. 2012, ApJS, 201, 19

Epchtein, N., de Batz, B., Capoani, L., et al. 1997, The Messenger, 87, 27

ESA 1997, HIPPARcos and Tycho catalogues, ESA-SP 1200

Folkes, S. L., Pinfield, D. J., Jones, H. R. A., et al. 2012, MNRAS, 427, 3280

Gelino, C. R., Kirkpatrick, J. D., \& Burgasser, A. J. 2012, online database for $1281 \mathrm{~L}$ and T dwarfs at DwarfArchives.org (status: 6 November 2012) Gudehus, D. H. 2001, BAAS, 33, 850

Hambly, N. C., MacGillivray, H. T., Read, M. A., et al. 2001, MNRAS, 326, 1279

Hambly, N. C., Henry, T. J., Subasavage, J. P., Brown, M. A., \& Jao, W.-C. 2004, AJ, 128, 437

Henry, T. J., Ianna, P. A., Kirkpatrick, J. D., \& Jahreiß, H. 1997, AJ, 114, 388

Henry, T. J., Subasavage, J. P., Brown, M. A., et al. 2004, AJ, 128, 2460

Jao, W.-C., Henry, T. J., Subasavage, J. P., et al. 2005, AJ, 129, 1954

Kirkpatrick, J. D., Reid, I. N., Liebert, J., et al. 1999, ApJ, 519, 802

Kirkpatrick, J. D., Cushing, M. C., Gelino, C. R., et al. 2011, ApJS, 197, 19

Kirkpatrick, J. D., Gelino, C. R., Cushing, M. C., et al. 2012, ApJ, 753, 156

Knapp, G. R., Leggett, S. K., Fan, X., et al. 2004, AJ, 127, 3553

Lépine, S., Shara, M. M., \& Rich, R. M. 2002, AJ, 124, 1190

Lucas, P. W., Tinney, C. G., Burningham, B., et al. 2010, MNRAS, 408, L56

Luhman, K. L. 2013, ApJ, 767, L1

Looper, D. L., Kirkpatrick, J. D., Cutri, R. M., et al. 2008, ApJ, 686, 528

Monet, D. G., Levine, S. E., Canzian, B., et al. 2003, AJ, 125, 984

Parker, Q. A., Phillipps, S., Pierce, M. J., et al. 2005, MNRAS, 362, 689

Pavlenko, Y. V., Jones, H. R. A., Martín, E. L., et al. 2007, MNRAS, 380, 1285

Phan-Bao, N., Bessell, M. S., Martín, E. L., et al. 2008, MNRAS, 383, 831

Rebolo, R., Martín, E. L., \& Magazzu, A. 1992, ApJ, 389, L83

Ribas, I. 2003, A\&A, 400, 297

Röser, S., Demleitner, M., \& Schilbach, E. 2010, AJ, 139, 2440

Scholz, R.-D., Bihain, G., Schnurr, O., \& Storm, J. 2012, A\&A, 541, A163

Skrutskie, M. F., Cutri, R. M., Stiening, R., et al. 2006, AJ, 131, 1163

Tinney, C. G. 1996, MNRAS, 281, 644

Tinney, C. G. 1998, MNRAS, 296, L42

Tinney, C. G., \& Reid, I. N. 1998, MNRAS, 301, 1031

van Leeuwen, F. 2007, A\&A, 474, 653

Wright, E. L., Eisenhardt, P. R. M., Mainzer, A. K., et al. 2010, AJ, 140, 1868 